\title{
Adaptation of the Bangla Version of the COVID-19 Anxiety Scale
}

\author{
Oli Ahmed ${ }^{1}$ (D) Rajib Ahmed Faisal $^{2}$ (D) Tanima Sharker $^{3}$ (D) Sherman A. Lee $^{4}$. \\ Mary C. Jobe $^{5}$ iD
}

Published online: 27 June 2020

(C) Springer Science+Business Media, LLC, part of Springer Nature 2020

\begin{abstract}
The Coronavirus Anxiety Scale (CAS) is among the first few published screening tools for assessing dysfunctional anxiety induced by the current COVID-19 pandemic. The CAS was translated into the Bangla language following the International Test Commission's guidelines for this adaptation study and placed in an online survey $(N=737$, with a mean age of 26.55 ( $S D=7.166$ years) to assess the psychometric properties of the Bangla version of the scale. Results suggested that all items had a good item discrimination index and single-factor structure with good factor loadings. The CAS Bangla version was found to have good internal consistency reliabilities, test-retest reliability, and composite reliability ( $\geq 0.7$ ). The measurement invariance suggested invariances across age groups and gender. The CAS Bangla version showed a high correlation to the anxiety subscale of the short form of the Depression Anxiety Stress Scale (DASS-21) and a moderate correlation to the depression subscale of the DASS-21 and the COVID-19 Worry Scale. This
\end{abstract}

Rajib Ahmed Faisal

faisal.ier@cu.ac.bd

Oli Ahmed

oliahmed_polash131@cu.ac.bd

Tanima Sharker

tanima.bmb@nstu.edu.bd

Sherman A. Lee

sherman.lee@cnu.edu

Mary C. Jobe

mary.jobe.16@cnu.edu

1 Department of Psychology, University of Chittagong, Chittagong 4331, Bangladesh

2 Institute of Education and Research (IER), University of Chittagong, Chittagong 4331, Bangladesh

3 Department of Biochemistry and Molecular Biology, Noakhali Science and Technology University, Noakhali 3814, Bangladesh

4 Department of Psychology, Christopher Newport University, 1 University Place, Newport News, VA 23606, USA

5 Christopher Newport University, 1 University Place, Newport News, VA 23606, USA 
validation of a Bangla CAS scale would be helpful for mental health practitioners to assess pandemic anxiety among the Bangladeshi people.

Keywords Anxiety Anxiety scale $\cdot$ COVID-19 pandemic $\cdot$ Psychometric properties $\cdot$ Bangladesh

\section{Background}

The world is amid a global pandemic, causing instability in every area of human life. The situation is a genuinely frightening and daunting time for many. The discovery of the epidemic was first proclaimed on the last day of 2019 in Wuhan, China (World Health Organization 2020). In the months since, SARS-CoV-2 or COVID-19 is now the third deadliest pandemic to have faced the twenty-first century (Washington Post 2020; Worldometer 2020a, May 22). At this point, the rate of infection growth, causality, and recuperation is unknown (Sharma et al. 2020). So far, the novel virus has infected over five million $(5,199,016)$ people globally (Worldometer 2020b, May 22). The death rate of COVID-19 is rising fast across the world, driving many to become desperate for a solution. In addition, the unpredictability of the virus' nature creates an environment of ongoing stress, which can increase the risks of people developing psychological disorders (Zandifar and Badrfam 2020). These psychological aspects must be addressed as the mental strain will likely continue following the pandemic, as people will continue to grieve the deaths of those who they lost to the novel virus (Taylor and Asmundson 2020).

Moreover, in order to reduce the rapid spread of the virus, all affected countries have imposed full or partial lockdowns and instituted a range of practices (e.g., "social isolation," "selfisolation," "spatial distancing," "quarantine," etc.) to prevent people from gathering in large numbers. Although these first-line emergency responses are designed to reduce the spread of the infectious disease, many people who are quarantined may develop feelings of loneliness, boredom, physical inactivity, and insecurities about food and finances (Brooks et al. 2020; Kamal 2020; Seppo 2020). Although these routines are designed to limit people's exposure to the deadly virus, they can also induce fear and anxiety about infection for those who partake in these new practices (McKay et al. 2020). Recent studies on the psychological effects during the COVID-19 pandemic have suggested higher than usual rates of anxiety and depression in the population (Ahmed et al. 2020a; Wang et al. 2020). According to the Anxiety and Depression Association of America (ADAA 2020), the current outbreak of the coronavirus has caused increased anxiety levels among many in the public community. Recent studies have supported this claim, showing that fear and anxiety about COVID-19 are associated with higher anxiety and depression symptoms (Ahorsu et al. 2020; Lee et al. 2020). Given the importance of identifying coronavirus anxiety in the population, because of its negative influence on people's mental health and wellbeing during the pandemic, it is imperative that health professionals and researchers screen people for this condition. However, to date, there are no known instruments to assess dysfunctional coronavirus anxiety in Bangladesh. Thus, the purpose of this study is to validate the Coronavirus Anxiety Scale (CAS; Lee 2020a) as a clinical mental health screener in the Bangla language.

\section{Present Study}

At the time of writing, 30,205 people have tested positive for COVID-19, and 432 have died by the virus in Bangladesh (Institute of Epidemiology Disease Control and Research 2020, May 22). 
For the country, the number of positive cases and deaths are both still increasing. As a first-line emergency response to the COVID-19 outbreak, the Bangladesh government announced general leave and a shutdown of all offices, markets, transports, etc., except for emergency services, since March 26, 2020 (WION News 2020). Since shutdowns, Bangladeshi people have been isolated and living with fear and uncertainty over this prolonged period (Nooruddin and Shahid 2020). That aside, many are suffering from the psychological effects of this situation across the globe (Limcaoco et al. 2020; Wang et al. 2020); Bangladesh is no exception to that - seeing its first COVID-19 suicide case as a result of coronavirus fears (Mamun and Griffiths 2020). Therefore, it is vital that the Bangladeshi people who appear to be mentally affected be efficiently and effectively screened for psychological services as soon as possible. Thus, the present study aims to translate the CAS into the Bangla language so that its adaption can be used for the assessment of dysfunctional anxiety, due to the COVID-19 outbreak, for Bangladeshi people.

\section{Method}

\section{Participants}

An online survey was conducted to collect data using a survey link created on a Google Form. It was then distributed via email and social media like Facebook, WhatsApp, etc. The only inclusion criterion to participate in this study was that participants had to be at least 18 years old. A total of 737 people completed the online survey; among them, eight were excluded as they are below 18 years. The remaining participants' ages ranged from 18 to 99 years $(M=$ $26.55, S D=7.166$ ). Among the 729 participants, $60.6 \%$ were male, $39.4 \%$ were female, $2.6 \%$ completed secondary education, $14.7 \%$ completed higher secondary school, $46.5 \%$ had an undergraduate degree, and $35.8 \%$ had post-graduate degrees. Among them, $64.5 \%$ were living in urban areas, $15 \%$ in semi-urban areas, and $20.6 \%$ were in rural areas. In terms of employment status, $60.4 \%$ were students, $29.9 \%$ were full-time employees, $4.4 \%$ in business or self-employed, $3.3 \%$ in other jobs, and $2.4 \%$ were unemployed.

\section{Ethics}

The present study was conducted following the Declaration of Helsinki and its later amendments or comparable ethical standard. Additionally, the study received ethical approval, before the study was undertaken, from the Noakhali Science and Technology University (reference no. 21/2020). Study objectives, potential benefits, risks, the confidentiality of given responses, etc. were communicated with participants in an informed consent form, prior to starting the survey. After reading the form, participants gave their consent to participate by clicking either "I agree to participate" or "I don't agree to participate." Only after clicking on "I agree to participate" was a participant able to take part in the survey.

\section{Measures}

The online survey included the CAS (Lee 2020a), the COVID-19 Worry Scale (CWS; Ahmed et al. 2020b), the anxiety and depression subscales of a short form of the Depression Anxiety Stress Scale (DASS-21; Lovibond and Lovibond 1995; Alim et al. 2014 [Bangla version]), the brief Warwick-Edinburgh Mental Wellbeing Scale (SWEMWBS; Stewart-Brown et al. 2009; 
Rahman and Imran 2013 [Bangla version]), and demographic information including gender, age, residence status, education level, and employment status.

\section{Coronavirus Anxiety Scale}

The CAS (Lee 2020a) is a valid unidimensional tool that assesses the physiological reactions of fear and anxiety related to the COVID-19 pandemic. This dysfunctional coronavirus anxiety scale consists of 5 items (e.g., "I lost interest in eating when I thought about or was exposed to information about the coronavirus"). In addition, it has excellent internal consistency reliability, construct, and concurrent validity. For the scale, participants were asked to rate the items on a 5-point Likert-type scale, from 0 (not at all) to 4 (nearly every day over the last 2 weeks). The cutoff score for this scale is $\geq 9$, with $90 \%$ sensitivity and $85 \%$ specificity.

In the present study, the CAS was translated into Bangla following the guidelines of the International Test Commission (International Test Commission 2018) for the translation and adaptation of measurement instruments from one language to another language. At first, the CAS was translated into Bangla from English independently by two bilingual experts, and then, these two versions were synthesized into one. Next stage, the Bangla version of the CAS was back-translated into English by two other bilingual experts and then combined into one. The original English and back-translated versions were compared with check whether the meaning of any item differed. There was no significant difference in meaning between the original and backtranslated versions. In the next stage, the translated CAS was put into a pilot study $(n=22,54.5 \%$ female, mean age $=23.55(S D=1.30)$ years, and COVID-19 anxiety $M=3.32, S D=2.97)$ where a cognitive interview was taken. Results suggested that all participants understood all items of this version of the CAS clearly. Having no problems in the pilot study, the translated CAS was placed into a final test for assessing its psychometric properties. For the present study, the CAS Bangla version demonstrated good internal consistency $(\omega=0.879, \alpha=0.872)$.

\section{COVID-19 Worry Scale}

The CWS (Ahmed et al. 2020b) is a valid unidimensional tool for assessing worries related to the COVID-19 infection. This scale consists of 7 items (e.g., "How concerned are you about being affected by the Coronavirus?") that assess the concerns about self, family members, relatives, etc. being affected by COVID-19. Authors have reported that the CWS has good internal consistency reliabilities ( 0.81 to 0.85 ), construct (factor loadings ranging from 0.70 to 0.83 ), and convergent validity. The CWS scores significantly correlated with stress, anxiety, and depression scores of the DASS-21. Participants were asked to rate each item using a 4-point Likert-type scale, from 1 (not at all) to 4 (very much). Total scores could range from 7 to 28 . For this scale, respondents who scored 23 or above are considered highly worried. In the present study, the CWS showed good internal consistency $(\omega=.881, \alpha=.875)$.

\section{Short Depression Anxiety Stress Scale}

The Depression Anxiety Stress Scale (DASS; Lovibond and Lovibond 1995) is a valid tool for quick assessment of depression, anxiety, and stress. This scale has 42 items with 14 items per subscale. Participants were asked to rate their responses based on their experiences from the previous week. Items were rated on a 4-point Likert-type scale, from 0 (did not apply to me at all) to 3 (applied to me very much or most of the time). Total scores could range from 0 to 42 
for each subscale. The DASS has good internal consistency reliability and construct and concurrent validity. The shorter version of the DASS includes 21 items, with 7 items in each subscale. Antony et al. (1998) suggest using the DASS-21 since it has similar psychometric properties to the longer DASS-42. When scoring the DASS-21, the sum of the 7 items in each subscale needs to be multiplied by 2 to get a total score. The DASS-21 Bangla version (Alim et al. 2014) also has good psychometric properties for quick assessment of stress, anxiety, and depression among Bangladeshi people. For this study, the depression and anxiety subscales of the DASS-21 were used, and both subscales showed good internal consistency reliabilities ( $\omega=0.874, \alpha=0.872$ and $\omega=0.804, \alpha=0.899$, respectively).

\section{Short Warwick-Edinburgh Mental Wellbeing Scale}

The Warwick Edinburgh Mental Wellbeing Scale (WEMWBS; Tennant et al. 2007) is a 14item quick measure for assessing mental well-being among the general population. This scale along with the Bangla version (Rahman and Imran 2013) has good reliabilities and construct and concurrent validities. For the scale, participants were asked to rate their responses from their feelings over the past week. They were rated on a 5-point Likert-type scale, from 1 (none of the time) to 5 (all the time) - with total scores ranging from 14 to 70 . Using Rasch modeling, Stewart-Brown et al. (2009) suggest using the shorter version of the scale (SWEMWBS), consisting of only 7 items. Thus, for this study, we used the SWEMWBS as it showed superior interval scaling than the full-length scale. This shorter scale also showed good internal consistency reliability $(\omega=.876, \alpha=.874)$.

\section{Data Analysis}

The use of descriptive statistics (frequency, percentages, mean, and standard deviation) was used to assess the participants' characteristics from the study. Psychometric properties of the CAS Bangla were assessed using the classical test theory (CTT). Under the CTT, we assessed corrected item-total correlation (minimum of .30; Field 2017), internal consistency reliabilities (Cronbach's alpha, McDonald's omega, and using the Spearman-Brown formula; accepted value $\geq 0.70$ ), standard error of measurement (accepted value $<S D / 2$; Wuang et al. 2012), average variance extracted (accepted values $\geq 0.50$ ), composite reliability (accepted values $\geq$ 0.70 ), and standard error of measurement (accepted value $<S D / 2$; Wuang et al. 2012).

Confirmatory factor analysis (CFA) was performed to assess the factor structure of the CAS Bangla version. Using CFA, the unidimensional factor structure suggested by the author was tested. Model fits were assessed through the $\chi^{2} / d f$ value, comparative fit index (CFI), goodnessof-fit index (GFI), Tucker-Lewis index (TLI), root mean square error of approximation (RMSEA), and standardized root mean square residual (SRMR). The $\chi^{2} / d f$ ratio value $\leq 2$ suggests a good fit (Schermelleh-Engel and Moosbrugger 2003). However, Marsh and Hocevar (1988) suggested that this value should be less than 5. The CFI, GFI, and TLI values $\geq 0.95$ suggest the good fit model (Hu and Bentler 1999). Additionally, a RMSEA value between 0.06 and 0.08 and a $S R M R$ value $\leq 0.08$ (Schreiber et al. 2006) suggest a better-fitted model.

Next, a series of multigroup CFA was performed to assess the measurement invariance of the CAS Bangla across demographic groups. In the present study, measurement invariance was assessed across age groups (18 to 29 years vs. 30 years and above) and gender (male vs. female). There were three models in multigroup CFA for each demographic-model 1 (configural invariance model: same structure across the groups), model 2 (metric model: factor loadings 
constrained equal across the groups), and model 3 (scalar model: factor loading and intercepts constrained similarly across the groups). A non-significant $\Delta \chi^{2}$ value suggests measurement invariance across the groups. As the $\Delta \chi^{2}$ is an excessive stringent test of invariance (MacCallum et al. 1992) and sensitive to sample size (Dimitrov 2010), in this study we used $\Delta C F I$, $\triangle \mathrm{RMSEA}$, and $\triangle$ SRMR to make decisions concerning invariance. Chen (2007) recommended $\Delta \mathrm{CFI} \geq 0.010, \Delta \mathrm{RMSEA} \leq 0.015$, and $\Delta \mathrm{SRMR} \leq 0.01$ as evidence of measurement invariance.

Besides the above described statistical tests, the Pearson product-moment correlation coefficient was also performed to assess the convergent validity of the CAS Bangla version.

\section{Results}

Table 1 shows the mean and standard deviation for each item, ranging between $0.28(0.71)$ and 0.86 (1.12). Table 1 also shows that all items have good corrected item-total correlations ranging from 0.68 (item 5) to 0.73 (item 4). The CAS Bangla version also has good internal consistency reliabilities (Cronbach's alpha, 0.872; McDonald's omega, 0.879; and split half reliability through the Spearman-Brown formula, 0.864; Table 2). The single-factor model in the CFA yielded a good model fit $(\mathrm{GFI}=0.987, \mathrm{CFI}=0.989, \mathrm{TLI}=0.977$, $\mathrm{RMSEA}=0.074$, and SRMR $=0.019$ ) except $\chi^{2} / d f$ ratio (5.001). Modification indices were examined, and the model was modified through adding a covariance between error variances for item 1 and item 4. All indices of the modified model yielded good model fit $\left(\chi^{2} / d f=2.331, \mathrm{GFI}=0.995, \mathrm{CFI}=\right.$ $0.997, \mathrm{TLI}=0.992, \mathrm{RMSEA}=0.043$, and $\mathrm{SRMR}=0.013$ ). Factor loadings ranged from 0.72 (item 5) to 0.83 (item 4). The CAS Bangla version also demonstrated acceptable test-retest reliability $(r=0.764, p<0.001 ; 95 \%$ CI $0.522,0.893)$, average variance extracted $(0.601)$, composite reliability (0.882), and standard error of measurement $(1.479<S D / 2$ [2.067]).

Results (Table 3) of the multigroup analysis demonstrated that there was a metric and scalar invariance between the two age groups. The configural model had good model fit $\left(\chi^{2} / d f=\right.$ $4.105, \mathrm{CFI}=0.986, \mathrm{RMSEA}=0.065$, and SRMR $=0.0214$ ). There were negligible changes in CFI, RMSEA, and SRMR between configural and metric models $(0.002,-0.010,0.0042$, respectively) and between metric and scalar models $(0.003,-0.004,-0.0001$, respectively). Results (Table 3) regarding invariance across gender suggest full metric invariance and partial scalar invariance between males and females. Moreover, the configural model of gender had good model fit $\left(\chi^{2} / d f=1.809, \mathrm{CFI}=0.996, \mathrm{RMSEA}=0.033\right.$, and $\left.\mathrm{SRMR}=0.0164\right)$. Although there were negligible changes in CFI, RMSEA, and SRMR values between configural and metric models $(0.003,002,0.0064$, respectively), notable changes were in the CFI and RMSEA values between metric and scalar models (0.013 and 0.017). Item 4 was freed in the scalar model and showed little changes in CFI and RMSEA values from metric to scalar

Table 1 Descriptive and psychometric properties of the Coronavirus Anxiety Scale at the item level

\begin{tabular}{lllll}
\hline Item & $M$ & $S D$ & Corrected item-total correlation & Factor weight \\
\hline Item 1 & 0.78 & 1.15 & 0.70 & 0.79 \\
Item 2 & 0.86 & 1.12 & 0.71 & 0.76 \\
Item 3 & 0.71 & 1.05 & 0.72 & 0.78 \\
Item 4 & 0.63 & 1.00 & 0.73 & 0.83 \\
Item 5 & 0.28 & 0.71 & 0.68 & 0.72 \\
\hline
\end{tabular}

$M$ mean; $S D$ standard deviation 
Table 2 Psychometric properties of the Coronavirus Anxiety Scale at the scale level

\begin{tabular}{lll}
\hline Psychometric properties & Value & Suggested cutoff \\
\hline Cronbach's alpha & 0.872 & $\geq 0.70$ \\
McDonald's omega & 0.879 & $\geq 0.70$ \\
Split-half reliability (Spearman-Brown formula) & 0.864 & $\geq 0.70$ \\
Test-retest reliability & 0.764 & $\geq 0.70$ \\
AVE & 0.601 & $\geq 0.50$ \\
CR & 0.882 & $\geq 0.70$ \\
SEM & 1.479 & $<D / 2$ \\
Model fits of confirmatory factor analysis & & \\
$\chi^{2}(d f, p$ value) & $9.324(4,0.053)$ & $n s$ \\
$\chi^{2} / d f$ & 2.331 & $<5$ \\
GFI & 0.995 & $>0.95$ \\
CFI & 0.997 & $>0.95$ \\
TLI & 0.992 & $>0.95$ \\
RMSEA & 0.043 & $<0.08$ \\
SRMR & 0.013 & $<0.08$ \\
\hline
\end{tabular}

AVE, average variance extracted; CR, composite reliability; SEM, standard error of measurement; SD, standard deviation; df, degrees of freedom; ns, nonsignificant; GFI, goodness of fit index; CFI, comparative fit index; TLI, Tucker-Lewis index; RMSEA, root mean square error of approximation; SRMR, standardized root mean square residual

models (0.006 and 0.008). Although there was a difference in mean and intercept in item 4 between males and females, the CAS Bangla is a valid measure for both groups.

Total scores of the CAS Bangla version correlated strongly and positively to COVID-19 worry, anxiety, depression, and negatively to mental well-being (Table 4); this suggests the concurrent validity of the CAS Bangla version. The scale's cutoff score parameters were based on the CAS' original findings (Lee 2020a) and not calculated for this sample. However, to determine differences between groups, comparisons were made across gender, residence, and education statuses. An independent sample $t$ test result showed a significant mean difference $(t(727)=4.25, p<0.001 ; d=-0.32)$ between males $(M=2.76, S D=3.90)$ and females $(M=$ 4.07, $S D=4.36)$. Additionally, a one-way ANOVA suggested non-significant mean differences in residence status $(F(2726)=0.020, p=0.980)$ and education level $(F(3722)=0.993$, $p=0.396)$. Observations for below secondary education and Ph.D. degree educational levels were excluded from this analysis due to the fewer number of them.

Table 3 Measurement invariances of the Coronavirus Anxiety Scale across age and gender groups

\begin{tabular}{lcccccccccr}
\hline Model & $\chi^{2}$ & $d f$ & $\Delta$ & $p$ value & CFI & $\Delta$ CFI & RMSEA & $\Delta$ RMSEA & SRMR & $\Delta$ SRMR \\
\hline Age group (18 to 29 vs. 30 and older) & & & & & & & \\
Configural & 32.843 & 8 & & & 0.986 & & 0.065 & & 0.0214 & \\
Metric & 41.281 & 13 & 8.438 & 0.13 & 0.984 & 0.002 & 0.055 & -0.010 & 0.0256 & 0.0042 \\
Scalar & 52.448 & 18 & 11.167 & 0.05 & 0.981 & 0.003 & 0.051 & -0.004 & 0.0255 & -0.0001 \\
Gender (male vs. female) & & & & & & & & & \\
Configural & 14.476 & 8 & & & 0.996 & & 0.033 & & 0.0164 & \\
Metric & 24.618 & 13 & 10.142 & 0.07 & 0.993 & 0.003 & 0.035 & 0.002 & 0.0228 & 0.0064 \\
Scalar & 53.230 & 18 & 28.612 & $<0.01$ & 0.980 & 0.013 & 0.052 & 0.017 & 0.0259 & 0.0031 \\
Partial scalar & 40.152 & 17 & 15.534 & $<0.01$ & 0.987 & 0.006 & 0.043 & 0.008 & 0.0251 & 0.0023 \\
\hline
\end{tabular}

df, degrees of freedom; CFI, comparative fit index; RMSEA, root mean square error of approximation; SRMR, standardized root mean square residual 
Table 4 Correlation of COVID-19 anxiety with COVID-19 worry, anxiety, depression, and mental well-being COVID-19 anxiety

\begin{tabular}{lrl}
\hline & $r$ & $95 \%$ CI \\
\hline COVID-19 worry & $0.390^{*}$ & {$[0.327,0.450]$} \\
Anxiety & $0.653^{*}$ & {$[0.609,0.693]$} \\
Depression & $0.516^{*}$ & {$[0.460,0.567]$} \\
Mental well-being & $-0.238^{*}$ & {$[-0.305,-0.168]$} \\
\hline
\end{tabular}

$r$ Pearson product-moment correlation coefficient; CI, confidence interval

$* p<0.001$

\section{Discussion}

The COVID-19 pandemic is threatening for the physical as well as mental health of individuals (Wang et al. 2020). To understand the impact this pandemic has on individuals - scientists, psychologists, and researchers are working on developing different tools to assess both the physical and mental health of individuals. The CAS and the Fear of COVID-19 Scale (Ahorsu et al. 2020) are among the first few documented psychopathology-related tests for COVID-19 anxiety. The CAS is the first that was validated on a large sample of specifically US adults who reported severe anxiety in the early stages of the coronavirus pandemic, including a significant sample of people diagnosed with the disease (Lee 2020a). The present study was undertaken to adapt the CAS in the Bangla language to assess dysfunctional COVID-19 anxiety among Bangladeshi people. In this adaptation process of the CAS, data were collected via an online survey, and item and scale level psychometric properties were assessed.

Results show that the items of the CAS Bangla version had high item discrimination (itemtotal correlations). As a discrimination index, a good item-total correlation suggests that the corresponding item can discriminate between high and low scorers, in the test that the item belongs to. The higher item-total correlation of each item indicated that the items of the CAS Bangla version would be able to differentiate between low and high scorers, which are a strong psychometric feature of this instrument. Results regarding reliabilities show that the CAS Bangla version had good internal consistency reliabilities, test-retest reliability, and composite reliability $(\geq 0.7)$. In the original study and a replication analysis, Lee (2020a, b) found high internal consistency reliability for the CAS ( $\alpha$ s ranged between 0.92 and 0.93). Furr (2011) suggests 0.70 to 0.80 as sufficient reliability, although there are no clear cutoff values for poor and good reliabilities. However, others suggest 0.80 or higher reliability for screening purposes (Bardhoshi and Erford 2017; Erford 2013). As internal consistency reliabilities and composite reliability of the CAS Bangla version were higher than 0.80, this scale is suitable for screening COVID-19 anxiety among Bangladeshi people.

The CFA supported the single-factor model suggested by Lee $(2020 \mathrm{a}, \mathrm{b})$ studies. The single factor model of the Bangla CAS showed good model fits. The results regarding multigroup CFA suggest scalar invariance between ages 18 to 29 years and 30 years and older. Regarding gender, this scale had partial scalar invariance, as item 4 was variant between males and females. Aside from this one item, the CAS Bangla version assessed the same construct for both males and females. Lee (2020a) also showed measurement invariance between similar age groups and between genders. Results regarding correlations suggested the convergent validity of the scale as it was highly correlated to the anxiety score of the DASS-21 subscale and moderately correlated to the depression score of the DASS-21 subscale and the CWS. The moderate correlations with 
the depression and worry scores suggest that the CAS Bangla version assesses two different constructs. Results also showed females had higher COVID-19 anxiety scores than males. This finding is consistent with other studies that assessed general anxiety among people during the COVID-19 pandemic (Ahmed et al. 2020a; Wang et al. 2020). Altogether, our findings support the CAS Bangla version as a valid tool that exhibits appropriate psychometric properties in assessing dysfunctional coronavirus anxiety among the people of Bangladesh.

\section{Limitations}

The present study has several limitations. First, data were collected through a self-reported online survey that may have been subjected to the social desirability bias. Secondly, divergent validity was not assessed, as there were several online surveys when the data collection of this study was ongoing. From the authors' observations, people were reluctant to participate in a relatively longer survey. Therefore, the tool for assessing divergent validity was omitted from the questionnaire to keep participants' reports valid and complete. Future studies should use external validators to address this problem. Thirdly, data were collected using a non-clinical sample. Thus, a clinical sample should be used in further studies to validate the scale for clinical use.

\section{Recommendations}

Despite the above limitations, the CAS Bangla version has excellent psychometric properties to assess dysfunctional COVID-19 anxiety among Bangladeshi people. As this scale provides a quick assessment of anxiety related to the COVID-19 pandemic, it would be helpful for health professionals to address the general public's mental health issues caused by COVID-19, using this screening instrument. A part from the aforementioned recommendations of using external validators and validating the scale for clinical use, a further recommendation includes having the CAS Bangla version be used to treat anxiety via the delivery of an online psychotherapy - as not only has such telehealth therapy had a proven success rate (Khatri et al. 2014), but its online form may be a more practical solution during this pandemic.

\section{Conclusion}

The CAS, developed by Lee (2020a), was the first psychopathology related screening tool for COVID-19 anxiety on a US population; additionally, it is among the first few published scales to assess its construct. This study aimed to validate a Bangla version of the CAS to increase the reach of assessing those affected by this worldwide pandemic. After conducting an online study and various analyses, our results supported the CAS to be a valid instrument for assessing dysfunctional coronavirus anxiety for the Bangladeshi general population. With the CAS Bangla version's validation, those affected psychologically by the pandemic can be effectively screened in a quick fashion by both mental and healthcare professionals in Bangladesh. Finally, further research should explore our recommendations, such as examining the CAS Bangla version on a clinical sample as well as its treatment for COVID-19 anxiety via an online psychotherapy.

Funding information This study was done with self-funding. 
Compliance with Ethical Standards The present study was conducted following the Declaration of Helsinki and its later amendments or comparable ethical standard. Additionally, the study received ethical approval, before the study was undertaken, from the Noakhali Science and Technology University (reference no. 21/2020). Study objectives, potential benefits, risks, the confidentiality of given responses, etc. were communicated with participants in an informed consent form, prior to starting the survey.

Conflict of Interest The authors declare that they have no conflict of interest.

\section{Appendix}

\section{Bangla version of the Coronavirus Anxiety Scale (CAS)}

Table 5

অনুগ্রহ করে গত দুই সপ্তাহের কতটা সময় আপনার সাথে এমন হয়েছে তা বিবেচনা করে আপনার সাথে যে অপশনটি সবচেয়ে বেশি মিলে যায় সেটি নির্ধারণ করুন

\begin{tabular}{|c|c|c|c|c|c|c|}
\hline & & $\begin{array}{c}\text { কোনদিনই } \\
\text { না }\end{array}$ & $\begin{array}{l}\text { ১/২ } \\
\text { দিনের } \\
\text { কম }\end{array}$ & কয়েকদিন & $\begin{array}{l}\text { ৭ দিনের } \\
\text { বেশি সময় }\end{array}$ & $\begin{array}{l}\text { প্রায় } \\
\text { প্রতিদিন }\end{array}$ \\
\hline 1. & $\begin{array}{l}\text { করোনাভাইরাস } \\
\text { সম্পর্কিত খবর পড়লে } \\
\text { বা শুনলে আমার মাথা } \\
\text { ঝিমঝিম করে, মাথা } \\
\text { হালকা, অথবা অজ্ঞান } \\
\text { হওয়ার মতো অনুভুতি } \\
\text { হয় }\end{array}$ & 0 & 1 & 2 & 3 & 4 \\
\hline 2. & $\begin{array}{l}\text { করোনাভাইরাস সম্পর্কে } \\
\text { ভাবছিলাম বলে আমার } \\
\text { ঘুমাতে বা লম্বা সময় } \\
\text { ঘুমিয়ে থাকতে সমস্যা } \\
\text { হচ্ছিল }\end{array}$ & 0 & 1 & 2 & 3 & 4 \\
\hline 3. & $\begin{array}{l}\text { করোনাভাইরাস } \\
\text { চিন্তা করয় } \\
\text { বিষয়ে কোন বি এই } \\
\text { শুনলে/দেখলে আমার } \\
\text { শরীর অবশ বা } \\
\text { হিমশীতল হয়ে আসছিল }\end{array}$ & 0 & 1 & 2 & 3 & 4 \\
\hline 4. & $\begin{array}{lr}\text { করোনাভাইরাস } & \text { নিয়ে } \\
\text { চিন্তা করলে বা এই } \\
\text { বিষয়ে কোন } \\
\text { শুনলে/দেখলে } \\
\text { খাওয়াদাওয়ার আমি } \\
\text { হারিয়ে ফেলছিলাম }\end{array}$ & 0 & 1 & 2 & 3 & 4 \\
\hline 5. & $\begin{array}{l}\text { করোনাভাইরাস নিয়ে } \\
\text { চিন্তা করলে বা এই } \\
\text { বিষয়ে কোন কিছু } \\
\text { শুনলে/দেখলে আমার } \\
\text { বমির উদ্রেক বা পেটে } \\
\text { সমস্যা হচ্ছিল }\end{array}$ & 0 & 1 & 2 & 3 & 4 \\
\hline & মোট কলাম & + & + & + & + & + \\
\hline & & & & & \multicolumn{2}{|c|}{ সম্পূর্ণ ফলাফল } \\
\hline
\end{tabular}




\section{References}

ADAA. (2020). Coronavirus anxiety-Helpful expert tips and resources. Anxiety and Depression Association of America, ADAA. Retrieved May 22, 2020 from https://adaa.org/finding-help/coronavirus-anxiety-helpfulresources

Ahmed, M. Z., Ahmed, O., Aibao, Z., Hanbin, S., Siyu, L., \& Ahmad, A. (2020a). Epidemic of COVID-19 in China and associated psychological problems. Asian Journal of Psychiatry, 51, 102092. https://doi. org/10.1016/j.ajp.2020.102092.

Ahmed, M. Z., Ahmed, O., Alim, S., Khan, M. A. U., \& Rahman, S. (2020b). Covid-19 pandemic related psychological problems among Bangladeshi people. Manuscript submitted for publication.

Ahorsu, D. K., Lin, C.-Y., Imani, V., Saffari, M., Griffiths, M. D., \& Pakpour, A. H. (2020). The Fear of COVID19 Scale: Development and initial validation. International Journal of Mental Health and Addiction. https://doi.org/10.1007/s11469-020-00270-8.

Alim, S., Mahbub-E-Kibria, S., Islam, M., Uddin, M., Nessa, M., Wahab, M., \& Islam, M. (2014). Translation of DASS-21 into Bangla and validation among medical students. Bangladesh Journal of Psychiatry, 28(2), 6770.

Antony, M. M., Bieling, P. J., Cox, B. J., Enns, M. W., \& Swinson, R. P. (1998). Psychometric properties of the 42-item and 21-item versions of the Depression Anxiety Stress Scales in clinical groups and a community sample. Psychological Assessment, 10(2), 176-181. https://doi.org/10.1037/1040-3590.10.2.176.

Bardhoshi, G., \& Erford, B. T. (2017). Processes and procedures for estimating score reliability and precision. Measurement and Evaluation in Counseling and Development, 50(4), 256-263. https://doi.org/10.1080 /07481756.2017.1388680.

Brooks, S. K., Webster, R. K., Smith, L. E., Woodland, L., Wessely, S., Greenberg, N., \& Rubin, G. J. (2020). The psychological impact of quarantine and how to reduce it: rapid review of the evidence. The Lancet, 395, 912-920. https://doi.org/10.1016/S0140-6736(20)30460-8.

Chen, F. F. (2007). Sensitivity of goodness of fit indexes to lack of measurement invariance. Structural Equation Modeling: A Multidisciplinary Journal, 14(3), 464-504. https://doi.org/10.1080/10705510701301834.

Dimitrov, D. M. (2010). Testing for factorial invariance in the context of construct validation. Measurement and Evaluation in Counseling and Development, 43(2), 121-149. https://doi.org/10.1177/0748175610373459.

Erford, B. T. (2013). Assessment for counselors (2nd ed.) Cengage Wadsworth.

Field, A. (2017). Discovering statistics using SPSS (5th ed.). Sage Publications.

Furr, R. M. (2011). Scale construction and psychometrics for social and personality psychology. Sage Publications.

Hu, L., \& Bentler, P. M. (1999). Cutoff criteria for fit indexes in covariance structure analysis: Conventional criteria versus new alternatives. Structural Equation Modeling: A Multidisciplinary Journal, 6(1), 1-55. https://doi.org/10.1080/10705519909540118.

Institute of Epidemiology Disease Control and Research. (2020). Institute of Epidemiology Disease Control and Research. Bangladesh Covid-19 Update. https://www.iedcr.gov.bd/.

International Test Commission. (2018). ITC guidelines for translating and adapting tests (second edition). International Journal of Testing, 18(2), 101-134. https://doi.org/10.1080/15305058.2017.1398166.

Kamal, S. M. (2020). How Bangladesh is addressing the Covid19 pandemic. Observer Research Foundation. https:/www.orfonline.org/expert-speak/how-bangladesh-is-addressing-the-covid19-pandemic-65601/

Khatri, N., Marziali, E., Tchernikov, I., \& Shepherd, N. (2014). Comparing telehealth-based and clinic-based group cognitive behavioral therapy for adults with depression and anxiety: A pilot study. Clinical Interventions in Aging, 9, 765-770. https://doi.org/10.2147/CIA.S57832.

Lee, S. A. (2020a). Coronavirus anxiety scale: A brief mental health screener for COVID-19 related anxiety. Death Studies, 44(7), 393-401. https://doi.org/10.1080/07481187.2020.1748481.

Lee, S. A. (2020b). Replication analysis of the coronavirus anxiety scale. Dusunen Adam: The Journal of Psychiatry and Neurological Sciences, 33. https://doi.org/10.14744/DAJPNS.2020.00079

Lee, S. A., Jobe, M. C., \& Mathis, A. A. (2020). Mental health characteristics associated with dysfunctional coronavirus anxiety. Psychological Medicine, 1-2. https://doi.org/10.1017/S003329172000121X.

Limcaoco, R. S. G., Mateos, E. M., Fernandez, J. M., \& Roncero, C. (2020). Anxiety, worry and perceived stress in the world due to the COVID-19 pandemic, March 2020. Preliminary results. medRxiv. https://oi. org/10.1101/2020.04.03.20043992.

Lovibond, P. F., \& Lovibond, S. H. (1995). Manual for the depression anxiety stress scales (2nd ed.) Sydney Psychology Foundation.

MacCallum, R. C., Roznowski, M., \& Necowitz, L. B. (1992). Model modifications in covariance structure analysis: The problem of capitalization on chance. Psychological Bulletin, 111(3), 490-504. https://oi. org/10.1037/0033-2909.111.3.490. 
Mamun, M. A., \& Griffiths, M. D. (2020). First COVID-19 suicide case in Bangladesh due to fear of COVID-19 and xenophobia: Possible suicide prevention strategies. Asian Journal of Psychiatry, 51, 102073. https://oi. org/10.1016/j.ajp.2020.102073.

Marsh, H. W., \& Hocevar, D. (1988). A new, more powerful approach to multitrait-multimethod analyses: Application of second-order confirmatory factor analysis. Journal of Applied Psychology, 73(1), $107-117$. https://doi.org/10.1037/0021-9010.73.1.107.

McKay, D., Yang, H., Elhai, J., \& Asmundson, G. (2020). Anxiety regarding contracting COVID-19 related to interoceptive anxiety sensations: The moderating role of disgust propensity and sensitivity. Journal of Anxiety Disorders, 102233. https://doi.org/10.1016/j.janxdis.2020.102233.

Nooruddin, I., \& Shahid, R. (2020). Defusing Bangladesh's COVID-19 time bomb. Atlantic Council. https://www.atlanticcouncil.org/blogs/new-atlanticist/defusing-bangladeshs-covid-19-timebomb/.

Rahman, S., \& Imran, M. (2013). Bangladeshi adaptation of Warwick Edinburgh Mental Well Being Scale. Dhaka University Journal of Psychology, 37, 49-60.

Schermelleh-Engel, K., \& Moosbrugger, H. (2003). Evaluating the fit of structural equation models: Tests of significance and descriptive goodness-of-fit measures. Methods of Psychological Research Online, 8(2), 2374.

Schreiber, J. B., Nora, A., Stage, F. K., Barlow, E. A., \& King, J. (2006). Reporting structural equation modeling and confirmatory factor analysis results: A review. The Journal of Educational Research, 99(6), 323-338. https://doi.org/10.3200/JOER.99.6.323-338.

Seppo, M. (2020). From bustling streets to lockdown: Bangladesh and the UN mobilize to fight COVID-19: A UN resident coordinator blog. UN News. https://news.un.org/en/story/2020/04/1062552

Sharma, S., Sharma, M., \& Singh, G. (2020). A chaotic and stressed environment for 2019-nCoV suspected, infected and other people in India: Fear of mass destruction and causality. Asian Journal of Psychiatry, 51, 102049. https://doi.org/10.1016/j.ajp.2020.102049.

Stewart-Brown, S., Tennant, A., Tennant, R., Platt, S., Parkinson, J., \& Weich, S. (2009). Internal construct validity of the Warwick-Edinburgh Mental Well-being Scale (WEMWBS): A Rasch analysis using data from the Scottish health education population survey. Health and Quality of Life Outcomes, 7(1), 15. https://doi.org/10.1186/1477-7525-7-15.

Taylor, S., \& Asmundson, G. J. G. (2020). Life in a post-pandemic world: What to expect of anxiety-related conditions and their treatment. Journal of Anxiety Disorders, 72, 102231. https://doi.org/10.1016/j. janxdis.2020.102231.

Tennant, R., Hiller, L., Fishwick, R., Platt, S., Joseph, S., Weich, S., Parkinson, J., Secker, J., \& Stewart-Brown, S. (2007). The Warwick-Edinburgh Mental Well-being Scale (WEMWBS): Development and UK validation. Health and Quality of Life Outcomes, 5(1), 63. https://doi.org/10.1186/1477-7525-5-63.

Wang, C., Pan, R., Wan, X., Tan, Y., Xu, L., Ho, C. S., \& Ho, R. C. (2020). Immediate psychological responses and associated factors during the initial stage of the 2019 Coronavirus Disease (COVID-19) epidemic among the general population in China. International Journal of Environmental Research and Public Health, 17(5), 1729. https://doi.org/10.3390/ijerph17051729.

Washington Post. (2020). History's deadliest pandemics, from ancient Rome to modern America. https://www. washingtonpost.com/graphics/2020/local/retropolis/coronavirus-deadliest-pandemics/

WION News. (2020). COVID-19: Bangladesh will not extend lockdown after May 30. WION News. https://www.wionews.com/bangladesh/covid-19-bangladesh-will-not-extend-lockdown-after-may-30301544.

World Health Organization. (2020). Rolling updates on coronavirus disease (COVID-19): Events as they happen. World Health Organization https://www.who.int/emergencies/diseases/novel-coronavirus-2019/events-asthey-happen.

Worldometer. (2020a). Coronavirus death toll: 339,423 deaths. Retrieved May 22, 2020 from https://www. worldometers.info/coronavirus/coronavirus-death-toll.

Worldometer. (2020b). Coronavirus update (live): 5,173,838 cases and 333,173 deaths from COVID-19 virus pandemic - Worldometer. Retrieved May 22, 2020 from https://www.worldometers.info/coronavirus/.

Wuang, Y.-P., Su, C.-Y., \& Huang, M.-H. (2012). Psychometric comparisons of three measures for assessing motor functions in preschoolers with intellectual disabilities: Motor function measures for intellectual disabilities. Journal of Intellectual Disability Research, 56(6), 567-578. https://doi.org/10.1111/j.13652788.2011.01491.x.

Zandifar, A., \& Badrfam, R. (2020). Iranian mental health during the COVID-19 epidemic. Asian Journal of Psychiatry, 51, 101990. https://doi.org/10.1016/j.ajp.2020.101990.

Publisher's Note Springer Nature remains neutral with regard to jurisdictional claims in published maps and institutional affiliations. 